\title{
25 Research Soure \\ Quantification of the Impact of Factors Affecting the Technical Performance of Operating Room Personnel: Expert Judgment Approach
}

Khalil Taherzadeh Chenani ( $\sim$ khalil.oc.hy@gmail.com )

Shahid Sadoughi University of Medical Sciences and Health Services: Shahid Sadoughi University of Medical Sciences and Health Services

Reza Jafari Nodoushan

Shahid Sadoughi University of Medical Sciences and Health Services: Shahid Sadoughi University of Medical Sciences and Health Services

Mehdi Jahangiri

Shiraz University of Medical Sciences

Farzan Madadizadeh

Shahid Sadoughi University of Medical Sciences and Health Services

Hossein Fallah

Shahid Sadoughi University of Medical Sciences and Health Services

\section{Research}

Keywords: Human reliability, Healthcare, Surgical context, Performance shaping factors

Posted Date: May 26th, 2021

DOI: https://doi.org/10.21203/rs.3.rs-508736/v1

License: (c) (1) This work is licensed under a Creative Commons Attribution 4.0 International License. Read Full License 


\section{Abstract}

Background: Incorrect assessment of the performance shaping factors (PSFs), especially in healthcare systems may lead to irreversible consequences such as death. The aim of the current study was to adapt PSFs taxonomy given in standardized plant analysis risk human reliability analysis (SPAR-H) technique to surgical context.

Methods: Specialists from four teaching hospitals of Yazd city were participated in the current study using convenience sampling technique. PSFs taxonomy of the SPAR-H technique was revised and more context-specific definitions to surgical context were suggested. Step-wise weight assessment ratio analysis (SWARA) and eleven-digit numerical scale were used for quantification of the weight and negative influence rate of the PSFs during surgical processes, respectively. Probability density function (pdf) was used to specify the probability of the negative influence rate values, as well.

Results: Nine PSFs were proposed following the SPAR-H taxonomy. Order of the importance of the PSFs was quietly conflicting in terms of weight and negative influence rate. From the perspective of experts, fatigue and threat stress were assessed as the most important PSFs in terms of both weight and negative influence rate.

Conclusion: Current study has offered a domain-based understanding for the PSFs particularly in surgical context. Results of this study could be used to evaluate human error behaviors during the performance of high accuracy required tasks in operating room. Moreover, these results could be used in high-risk industries for improving patient safety programs in case of lacking literature in healthcare systems.

\section{Background}

Human error can adversely affect the safety, reliability of systems and equipment function and therefore may result in financial losses [1]. Throughout history, human factors have been cited as the reason behind many catastrophic events. More than $90 \%$ of nuclear industry accidents, $80 \%$ of chemical industry accidents, $75 \%$ of marine industry accidents and $70 \%$ of aviation industry events are caused by human factors [2]. In the study of catastrophic events reports, e.g. Bhopal (1984), Chernobyl (1986), Piper Alpha (1988) and Texaco Refinery (1994), human factors have been mentioned as one of the primary causes behind these events [3]. In literature, human factors are defined as the science of understanding human performance in a given system [4]. Human performance is also defined as the "measure of failures and actions under specified conditions" [5].

In healthcare sector, deficiencies in providing perfect patient safety and human error may lead to irreparable consequences like disability, disruption of health services, legal issues and even death [6]. One study indicates almost $5-10 \%$ of the patients have been effected by medical error, moreover, medical errors are responsible for more than 7,000 deaths annually in the United States [7]. In healthcare sector, human factors are defined as improving clinical performance by understanding the effects of teamwork, tasks, equipment, working environment, culture and organization on human behaviors and abilities 
through using that knowledge in clinical settings [4]. In healthcare sector, medical errors are practically always the result of human behavior in the environment where medical tasks should be performed [8].

Since the first stages of the emergence of human reliability assessment (HRA) approach, it was discovered that the technical performance of operators may be affected by two classes of factors: internal and external factors. The external factors are related to the environmental conditions where tasks should be performed, and the internal factors refer to the psychological and physiological conditions of the operators [9]. In various contexts, factors with identical concepts may be referred to with various titles and have different definitions. A more comprehensive definition of performance shaping factor (PSF) is as follows: "an aspect of the human's individual characteristics, environment, organization, or task that specifically decrements or improves human performance, thus respectively increasing or decreasing the likelihood of human error" [10].

It is worth noting that usage of empirical data to quantify the amount of intensity effect of the PSFs on the human error occurrence is considered as a substantial proceeding in HRA [11]. The lack of reliable data and extensive research about factors effecting individuals' technical performance in surgical setting, is considered as a fundamental problem in HRA in operating rooms. Considering this gap, adaptation of PSF taxonomies essentially designed for industrial setting to healthcare context seems to be necessary. The aim of the current study is to adapt PSFs taxonomy of SPAR-H technique to surgical context. And thus to suggest more explicit definitions for each PSF, assessment of the weight and the negative influence rate of the PSFs in surgical context using domain expert judgment approach.

\section{Materials And Methods}

\section{Study Design and Participants}

This cross-sectional and descriptive study was designed and carried out with the primary purpose of adapting new PSF taxonomy for surgical setting application following the SPAR-H technique (January and February, 2020). Operating rooms of four teaching hospitals of Yazd City were the sites where the present study was executed.

Based on the opinion of operating rooms management and personnel, surgeons, anesthesiologists, surgical technologists and anesthesiology technologists are four specialist groups who participate the most in operating processes. Because of this, we have conducted our study based on the judgments of these four specialist groups.

\section{Data collection procedure}

Convenience sampling technique was employed to recruit experts from operating rooms of the participating hospitals. Age, work experience (year), gender and specialty groups represented the considered demographic characteristics of the participated experts in this study. 
To capture experts' perception about the weight and the negative influence rate of the PSFs on their technical performance, a three-section questionnaire along with face-to-face interviews and interactive discussions, was employed. First section of the questionnaire was dedicated to the description of the purpose and the generalities of the whole study. Second and third sections were dedicated to the quantification of weight and negative influence rate of the PSFs, respectively.

\section{Suggested PSF Taxonomy Design}

Available Time, Stress/Stressors, Complexity, Experience/Training, Procedures, Ergonomics/ HMI, Fitness for duty and Work Processes form the PSF taxonomy of SPAR-H technique [12]. In the literature, deficiencies like unclear definitions and semantic overlap between PSFs that may lead to double calculation of the effect of a particular PSF are mentioned $[12,13]$. In the current study to overcome these deficiencies, more explicit definitions and new PSF taxonomy which are more consistent with surgical setting were suggested based on the PSF taxonomy of the SPAR-H technique.

Available time, threat stress, task complexity, experience/training, procedures, working conditions, humanmachine interface $(\mathrm{HMI})$, fatigue and teamwork are the nine PSFs of the suggested taxonomy for the surgical setting. Our suggested definitions for the PSFs are as follows:

Available Time: The purpose of the suggested definition for this PSF is to express the ratio between the time it takes to complete a specific task and the whole time available for completing it before any deleterious consequences emerge. Our suggested definition for the available time PSF follows Laumann and Rasmussen [14]: Available time represents the ratio between the time it takes to complete a specific task (required time) and the time available before the consequences of failing to complete the task are realized.

Threat Stress: In the definition of stress/stressors in the original SPAR-H guideline, there is some semantic overlap with other PSFs like Available time and Ergonomics/HMI [14]. It is stated that "one aspect of the stress/stressors PSF in SPAR-H that is not covered by other PSFs, the concept of threat stress" [14]. Our suggested definition for threat stress is: Threatening situation is when unstable conditions or novel environmental events may cause pain, discomfort or endanger patient's life. In this situation, operating room personnel are aware that their error may seriously endanger patient's life and have negative impact on their professional status.

Task Complexity: Complexity is defined as the amount of difficulty in task performance [12]. The SPAR-H guideline suggests 14 contributing factors to complexity. These factors considerably overlap with other PSFs, for example, "Transition between multiple procedures" overlaps with procedures PSF [12]. In the current study, the title task complexity is suggested for this PSF to present more objective definition that does not overlap with other PSFs and represents an expression of the complexity of the task in surgical context explicitly. Six categories of task complexity were suggested for the task complexity in one review study [15]: Goal complexity, Size complexity, Step complexity, Connection complexity, Dynamic complexity and Structure complexity. Our suggested definition follows these categories of task 
complexity [15]: Task complexity refers to the degree of complexity demanding for task performance according to the complexity categories. Which means the more complexity categories there are, the more complex the task is.

Experience/Training: In the SPAR-H guideline [12], this PSF specially refers to the experience and training of the operators involved in task performance. In the definition presented for this PSF, only the time of experience or training is considered. While other factors like the level, frequency and type of training that are more important in the success or failure of the task performance are overlooked [14]. Our suggested definition for Experience/training is: Experience/Training is related to possession or lack of task-related training, the frequency of task experiences in the field, the number of task-related retraining courses and the spent time between them.

Procedures: The concept of this PSF refers to the existence and use of work procedures to perform specific tasks [12]. In nuclear power industry, a procedure has been generally defined as "a written document (including both text and graphics) that presents a series of decision and action steps to be performed by plant personnel (e.g., operators, technicians) to accomplish a goal safely and efficiently" [16]. In the current study we have considered availability, quality and use of formal procedures in the suggested definition. Our suggested definition for the procedures PSF is: The procedure is a written document that provides a set of decision and action steps that must be followed by operating room personnel to safely and efficiently achieve task's goals. The purpose of procedures is to guide human actions when performing a task to reduce the likelihood of HEP and increase the chance for reaching task's goal efficiently.

Working conditions: In SPAR-H guideline, Ergonomics/HMI PSF is broadly defined and includes both aspects of $\mathrm{HMI}$ and Ergonomics [12]. In the current study we suggested the splitting of this PSF into two separate PSFs (working conditions and $\mathrm{HMI}$ ), to provide more clear definitions for these PSFs and to provide a chance of more accurate assessment of them in surgical context. Our suggested definition for working conditions PSF is: Working conditions PSF refers to the Physical elements of the working environment of operating room like noise, distraction, quality and quantity of lighting and temperature conditions.

Human-Machine Interaction (HMI): Laumann and Rasmussen recommended that HMI should encompass only the interactions between operators and computerized systems for reflecting the limited scope of this PSF [14]. Our suggested definition for HMI PSF is: The human machine interaction PSF encompasses the quality of equipment, displayers, annunciators, labeling, physical layout of the operating room, location of the operating room personnel during surgical processes and the adequacy or inadequacy of the information received from computerized systems for task performance.

Fatigue: In SPAR-H guideline [12], the definition of Fitness for duty PSF refers to the suitability of the individuals for task performance. One study has pointed out that Fatigue is much more cited in incidents reports than other indicators of fitness for duty PSF such as impairment due to drugs and alcohol usage, 
distraction and physical or mental capacity required for task performance [17]. In the current study we considered two types of Fatigue contributing factors, sleep homeostatic factors (overtime and sleep deprivation) and circadian factors (day or night shifts) [18]. Our suggested definition for fatigue PSF follows these contributing factors: Fatigue is related to the physical or mental exhaustion or sleepiness that is related to indicators such as sleep deprivation and day or night shifts.

Teamwork: Work processes defined poorly in the SPAR-H guideline [12], and indicators related to organization, safety culture, work planning, communication and teamwork are included in this PSF. Laumann and Rasmussen [14], suggested the splitting of this PSF. In the present study we focused on teamwork and defined it as a separate PSF. Our suggested definitions of team and teamwork are based on Salas et al. study [19]: A team is defined as "two or more individuals with specified roles interacting adaptively, interdependently, and dynamically toward a common and valued goal." Moreover, Teamwork is defined as "a set of interrelated thoughts, actions, and feelings of each team member that are needed to function as a team. And which are combined to facilitate coordinated, adaptive performance and task objectives resulting in value-added outcomes."

\section{Quantification of the PSFs Weight}

Step-wise weight assessment ratio analysis (SWARA) technique, as one of the multiple-criteria decisionmaking (MCDM) techniques, was used to determine the weight of each PSF (negative impact on technical performance of operating room personnel) during surgical processes in operating room [20].

\section{SWARA Technique:}

Experts should first arrange the criterions in order of importance. The most important criterion in human error occurrences is placed first and gets a score of one. Ultimately, the criterions are ranked based on the average values of their relative importance. The steps of this technique are as follows:

\section{- Criterion Arrangement}

Initially, the criterions should be arranged in order of importance. The most important criterions are arranged in the higher levels and the less important criterions arranged in the lower levels.

\section{- Comparative Importance of Average Value 0}

In this step, the relative importance of each criterion compared to the previous one is determined.

\section{- Calculation of the Coefficient}

Calculation of this coefficient is based on the relative importance of each criterion through Equation 1.

Equation 1:

$$
K_{j}=S_{j}+1
$$




\section{- Calculation of the Primary Weight of Each Criterion 0}

The primary weight of the criterion is calculated through Equation 2. In this regard, it should be noted that the weight of the first criterion, which is the most important criterion is considered 1.

Equation 2: $\quad Q_{j}=\frac{Q_{j}-1}{K_{j}}$

\section{- Calculation of the Final Weight of Each Criterion 0}

The final weight of each criterion, which also considered the normalized weight, is calculated through Equation 3.

Equation 3:

$$
W_{j}=\frac{Q_{j}}{\sum Q_{j}}
$$

\section{Quantification of the PSFs Influence Rate}

A numerical scale with eleven-digit (0-10) was used to evaluate the influence rate of each PSF ( 0 and 10 are the thresholds of the scale). Experts were asked to provide their subjective perception of how often each PSF has adversely influences their technical performance during surgical operations they have participated [21].

0 : the considered PSF has had no negative effect on the technical performance of operating room personnel at any surgical operation.

10: the considered PSF has had negative effect on the technical performance of operating room personnel at all of the surgical operations.

\section{Statistical Analysis}

Descriptive statistics, maximum (Max), minimum (Min), mean and median were used to investigate the quantitative and qualitative variables of demographic characteristics, respectively.

Statistical indicators, mean (Median), standard deviation (SD), Max and minimum Min were used to investigate the weights and the influence rates of each PSF. The order of weight and negative influence rate of the PSFs were expressed, as well in Table 1, 2.

Additionally, triangular distribution was used to investigate the probability density function of the PSFs. Triangular distribution frequently is used for the evaluation of epistemic uncertainties in risk analysis [22, 23]. "The triangular distribution contains three parameters: the lower limit a, the upper limit b, and the 
mode $\mathrm{m}$. The probability density at $\mathrm{x}=\mathrm{m}$ is determined by imposing the area of the triangle to 1" [21]. SPSS software version 23 was used for analyzing the gathered data.

\section{Results}

\section{Demographic Characteristics}

In total, 26 experts from four hospitals have participated in this study, there were 13 male and 13 female participants. The maximum and minimum age of the participants were 55 and 26 years (Mean $=32.6$, Median $=30$ ), respectively. In term of work experience (year), the maximum and minimum working experience of the experts were 30 and 4 (Mean $=8.8$, Median $=6$ ), respectively. Furthermore, the majority of the experts that took part in the survey were surgical technologists $(n=9)$ followed by anesthesiology technologists $(n=8)$, surgeons $(n=5)$ and anesthesiologists $(n=4)$.

\section{Weight And Influence Rate Of The Psfs}

Table 1 and Table 2 represent the descriptive statistics of the conducted SWARA for PSFs weight quantification and the negative influence rate of the PSFs, respectively.

In Table 1, the importance order represents PSFs order that more negatively effects the technical performance of operating room personnel. Which means, PSFs of 1 and 9 importance order have the most and least effect on the technical performance of the personnel, respectively.

According to Table 1, fatigue and procedures have the most and least negative effects on the technical performance of operating room staff, respectively. 
Table 1

Descriptive statistics of the weight of PSFs during surgical Processes

\begin{tabular}{|llllll|}
\hline PSFs & Mean (Median) & SD & Max & Min & Importance order \\
\hline Available time & $0.085(0.036)$ & 0.11 & 0.445 & 0.014 & 7 \\
\hline Threat Stress & $0.152(0.079)$ & 0.148 & 0.454 & 0.004 & 2 \\
\hline Task complexity & $0.105(0.066)$ & 0.123 & 0.436 & 0.003 & 6 \\
\hline Experience/Training & $0.127(0.071)$ & 0.145 & 0.465 & 0.005 & 4 \\
\hline Procedures & $0.06(0.037)$ & 0.064 & 0.245 & 0.005 & 9 \\
\hline Working conditions & $0.114(0.058)$ & 0.136 & 0.453 & 0.008 & 5 \\
\hline Human-Machine Interface (HMI) & $0.067(0.048)$ & 0.066 & 0.259 & 0.011 & 8 \\
\hline Fatigue & $0.16(0.147)$ & 0.146 & 0.452 & 0.011 & 1 \\
\hline Teamwork & $0.13(0.062)$ & 0.147 & 0.461 & 0.011 & 3 \\
\hline
\end{tabular}

As Table 2 shows, fatigue and threat stress have the most negative influence rate on the technical performance of operating room personnel. Influence rate order in Table 2, represents the order of the negative influence rate of the PSFs. Which means, PSFs of 1 and 9 have the most and least negative influence rate among others.

Table 2

Descriptive statistics of the negative influence rate of PSFs during surgical Processes

\begin{tabular}{|llllll|}
\hline PSFs & Mean (Median) & SD & Max & Min & Influence rate order \\
\hline Available time & $5.85(7)$ & 3.1 & 10 & 1 & 6 \\
\hline Threat stress & $6.88(7)$ & 2.47 & 10 & 2 & 2 \\
\hline Task Complexity & $6.54(6.5)$ & 2.49 & 10 & 3 & 3 \\
\hline Experience/Training & $6.4(6)$ & 2.51 & 10 & 1 & 4 \\
\hline Procedures & $5.58(5)$ & 2.11 & 10 & 1 & 7 \\
\hline Working conditions & $5.54(6)$ & 2.87 & 10 & 1 & 8 \\
\hline Human-Machine Interface (HMI) & $5.11(5)$ & 2.35 & 8 & 1 & 9 \\
\hline Fatigue & $7.77(8)$ & 2.1 & 10 & 3 & 1 \\
\hline Teamwork & $6.11(6)$ & 2.74 & 10 & 1 & 5 \\
\hline
\end{tabular}

\section{Probability Density Function Of The Psfs}


Accordingly, most likely value for each PSF has been calculated based on the observed values. Figure 1 describes the triangular probability distribution functions for the PSFs. As shown by Fig. 1, eight is the most likely value for available time PSF. Zero represents the most likely value for threat stress and fatigue PSFs. Nine is the most likely value for task complexity PSF. Four represents the most likely value for experience/training PSF. Five is the most likely value for teamwork, HMI and procedures PSF. And three represents the most likely value for working conditions PSF.

\section{Discussion}

This study was an attempt to explore the weights and the negative influence rates of the PSFs during surgical setting. In this regard, the results show fatigue and threat stress had the greatest impact on the operating room personnel technical performance during surgical processes in terms of weight and negative influence rate among others.

Fatigue had the most significant impact on the occurrence of human error (by weight of 0.16 ) and the first place of the negative influence rate (by negative influence rate of 7.77 ). One study has indicated that fragmented sleep or sleep deprivation causes decrement in reaction time, vigilance and cognitive processes [24]. In the current study, excessive weight and negative influence rate of fatigue compared to other PSFs show the necessity of the evaluation of fatigue as a separate PSF in HRA techniques especially in healthcare systems.

One study has evaluated the perioperative emotional stress as one of the influencing factors on technical performance of surgeons [21]. In current study, threat stress was determined as the PSF with the second most effect (by weight of 0.152 ) on the technical performance of operating room staff. Moreover, its negative influence rate also came in the second place (by negative influence rate of 6.88 ). Significant weight of threat stress may be attributed to the nature and high sensitivity of tasks performance in operating rooms and the anticipation of irreparable consequences in case of human error occurrences.

It is indicated that communication, one aspect of teamwork, as one of the major contributing factors to human error in intensive care units [25]. In the current study teamwork was determined as the third effective PSF (by weight of 0.13 ) and fifth one in term of negative influence rate (by negative influence rate of 6.11). One meta-analysis identified significant negative correlation between relationship conflicts and team performance [26]. Considering the results, it can be concluded that poor teamwork especially in highly sensitive environments or tasks with high need of participatory, like surgical processes, may leads to irreparable consequences like lifetime incapacitation or death.

It is indicated that deficiency in knowledge, skill and experience is the main cause of increasing the human error probability [27]. One review study has indicated that junior doctors are more prone to committing human errors [28]. In the current study, Experience/Training was determined as the forth effective PSF (by weight of 0.127 ), as well as, forth one in term of negative influence rate (by negative influence rate of 6.4). 
One study in healthcare context reported the share of $43 \%$ of medication errors to distraction [29]. In this study, working conditions determined as the fifth effective PSF (by weight of 0.114) and eighth one (by negative influence rate of 5.54) in term of negative influence rate. As it is obvious, working conditions should be considered as one of the PSFs that directly affects the technical performance of operating room personnel and may causes serious consequences.

Task complexity was determined as the sixth most effective PSF on the occurrence of human error (by weight of 0.105 ) and the third one in term of negative influence rate (by negative influence rate of 6.54). One study indicated that "Complex systems" had the third place among the most effective preliminary risk factors of the interaction between and hardware dimension in one maintenance activity [30].

Available time was determined as the seventh most effective PSF on the occurrence of human error (by weight of 0.085) and the sixth one in the term of negative influence rate (by negative influence rate of 5.85). One study weighted "Quick response is required because of time pressure" as the fifth most effective PSF in the mental load category in one maintenance activity [31]. High time pressure may cause operators to deal with high required cognitive process tasks in a time shortage situation, and this may have negative effects like anxiety increasing [32] and impairment of accuracy for dealing with perceptual tasks [33].

In three Mile Island accident report, deficiencies related to the HMI like lack of signals to show the level coolant and the opening of the pilot-operated relief valve are mentioned as the chief contributing factors [34]. HMI was determined as the eighth most effective PSF on the occurrence of human error (by weight of 0.067 ) and ninth in term of negative influence rate (by negative influence rate of 5.11). Problems related to the HMI PSF are mentioned among the primary causes of the industrial incidents. Whereas results of the present study indicate the insignificant importance of the HMI PSF in surgical context among others. And this may be attributed to the differences of the occupational contexts.

Procedures were determined as the ninth most effective PSF on the occurrence of human error (by weight of 0.06 ) and seventh one in term of negative influence rate (by negative influence rate of 5.58). Reports of various incidents have addressed procedure deficiencies as one of the major contributing factors, like Aberfan disaster (1966), Seveso incident (1976), Three Mile Island accident (1979) and Bhopal catastrophe (1984) [34]. In current study one of the most significant reasons that procedures PSF was considered as the least critical one among others, may be related to the fact that surgical teams are required to review surgical procedures and protocols before initiating the surgical process.

\section{Conclusions}

Most of the HRA techniques and their taxonomies, like SPAR-H have been developed for industrial settings. Application of such techniques and taxonomies for HRA in healthcare systems may be controversial in terms of tasks demands and results uncertainty. The suggested PSFs taxonomy and their quantified weights and negative influence rates, as well as, using probability density function for the 
perceived negative influence rate of PSFs, simplify determination of the most influencing PSFs on the technical performance of operating room personnel.

\section{Abbreviations}

PSFs: Performance shaping factors; SPAR-H: Standardized plant analysis risk human reliability analysis; SWARA: Step-wise weight assessment ratio analysis; pdf: Probability density function; HMI: Humanmachine interface; MCDM: multiple-criteria decision-making; Max: Maximum; Min: Minimum; SD: Standard deviation

\section{Declarations}

\section{Acknowledgements}

The authors would like to thank school of public health of shahid sadoughi university of medical sciences for their supports.

Authors' contributions

Reza Jafari Nodoushan: Study design, review of manuscript and editing of manuscript

Khalil Taherzadeh Chenani: Study design, Writing and editing of manuscript, data gathering and analysis Mehdi Jahangiri: Study design, review of manuscript and editing of manuscript

Farzan Madadizadeh: Data analysis, review and editing of manuscript

Hossein Fallah: review and editing of manuscript

\section{Funding}

This research received no specific grant from any funding agency in the public, commercial or not-forprofit sectors'.

\section{Availability of data and materials}

Anonymized datasets used and/or analyzed not including identifiable patient information are available from the corresponding author on reasonable request.

\section{Ethics approval and consent to participate}

Current study was approved by the ethics committee of school of public health of shahid sadoughi university of medical sciences with ethics code of IR.SSU.SPH.REC.1398.129. All participants provided informed consent. 


\section{Competing interests}

The authors declare that there is no conflict of interest.

\section{References}

1. Franciosi C, Di Pasquale V, lannone R, Miranda S. A taxonomy of performance shaping factors for human reliability analysis in industrial maintenance. J Ind Eng Manag. 2019;12(1):115-32. https://doi.org/10.3926/jiem.2702.

2. Jahangiri M, Hoboubi N, Rostamabadi A, Keshavarzi S, Hosseini AA. Human error analysis in a permit to work system: a case study in a chemical plant. Saf Health Work. 2016;7(1):6-11. https://doi.org/10.1016/j.shaw.2015.06.002.

3. Johnson C. Why human error modeling has failed to help systems development. Oxford University Press Oxford, UK. 1999;11(5):517 - 24. https://doi.org/10.1016/S0953-5438(98)00041-1.

4. Carthey J. Implementing human factors in healthcare. How to guide-volume 2. Taking further steps. Clinical Human Factors Group. 2013;2:1-59.

5. Dhillon BS. Human reliability, error, and human factors in power generation. Springer; 2014.

6. Ahmed Z, Saada M, Jones AM, Al-Hamid AM. Medical errors: Healthcare professionals' perspective at a tertiary hospital in Kuwait. PloS one. 2019;14(5):e0217023. https://doi.org/ 10.1371/journal.pone.0217023.

7. Küng K, Carrel T, Wittwer B, Engberg S, Zimmermann N, Schwendimann R. Medication errors in a swiss cardiovascular surgery department: a cross-sectional study based on a novel medication error report method. Nurs Res Pract. 2013;2013:671820. https://doi.org/ 10.1155/2013/671820.

8. Peters GA, Peters BJ. Medical error and patient safety: Human factors in medicine. CRC Press; 2007.

9. Pan X, Wu Z. Performance shaping factors in the human error probability modification of human reliability analysis. Int J Occup Saf Ergon. 2020;26(3):538-50. https://doi.org/ 10.1080/10803548.2018.1498655.

10. Boring RL, Blackman HS, editors. The origins of the SPAR-H method's performance shaping factor multipliers. 2007 IEEE 8th human factors and power plants and HPRCT 13th annual meeting; 2007: IEEE. https://doi.org/10.1109/HFPP.2007.4413202.

11. Kim Y, Park J, Jung W. A quantitative measure of fitness for duty and work processes for human reliability analysis. Reliab Eng Syst Saf. 2017;167:595-601. https://doi.org/10.1016/j.ress.2017.07.012.

12. Gertman D, Blackman H, Marble J, Byers J, Smith C. The SPAR-H human reliability analysis method. US Nuclear Regulatory Commission. 2005;230:35.

13. Groth KM, Mosleh A. A data-informed PIF hierarchy for model-based human reliability analysis. Reliab Eng Syst Saf. 2012;108:154 - 74. https://doi.org/10.1016/j.ress.2012.08.006. 
14. Laumann K, Rasmussen M. Suggested improvements to the definitions of Standardized Plant Analysis of Risk-Human Reliability Analysis (SPAR-H) performance shaping factors, their levels and multipliers and the nominal tasks. Reliab Eng Syst Saf. 2016;145:287-300. https://doi.org/10.1016/j.ress.2015.07.022.

15. Rasmussen M, Standal MI, Laumann K. Task complexity as a performance shaping factor: A review and recommendations in Standardized Plant Analysis Risk-Human Reliability Analysis (SPAR-H) adaption. Saf Sci. 2015;76:228-38. https://doi.org/10.1016/j.ssci.2015.03.005.

16. O'Hara JM, Higgins JC, Stibler WF, Kramer J. Computer-based procedure systems: technical basis and human factors review guidance. Brookhaven National Lab Upton Ny; 2000.

17. Whaley AM, Kelly DL, Boring RL, Galyean WJ. SPAR-H step-by-step guidance. Idaho National Laboratory (INL); 2012.

18. Liu P, Qiu Y, Hu J, Tong J, Zhao J, Li Z. Expert judgments for performance shaping factors' multiplier design in human reliability analysis. Reliab Eng Syst Saf. 2020;194:106343. https://doi.org/10.1016/j.ress.2018.12.022.

19. Salas E, Sims DE, Burke CS. Is there a "big five" in teamwork? Small Group Res. 2005;36(5):555-99. https://doi.org/10.1177/1046496405277134.

20. Keršuliene V, Zavadskas EK, Turskis Z. Selection of rational dispute resolution method by applying new step-wise weight assessment ratio analysis (SWARA). J Bus Econ Manag. 2010;11(2):243-58.

21. Onofrio R, Trucco P. Human reliability analysis (HRA) in surgery: Identification and assessment of Influencing Factors. Saf Sci. 2018;110:110-23. https://doi.org/10.1016/j.ssci.2018.08.004.

22. Johnson $D$. The triangular distribution as a proxy for the beta distribution in risk analysis. J R Stat Soc Ser D Stat. 1997;46(3):387-98. https://doi.org/10.1111/1467-9884.00091.

23. Kotz S, Van Dorp JR. A novel method for fitting unimodal continuous distributions on a bounded domain utilizing expert judgment estimates. IIE Trans. 2006;38(5):421-36. https://doi.org/10.1080/07408170500517267.

24. Krueger GP. Sustained work, fatigue, sleep loss and performance: A review of the issues. Work Stress. 1989;3(2):129-41. https://doi.org/10.1080/02678378908256939.

25. Donchin Y, Gopher D, Olin M, Badihi Y, Biesky MR, Sprung CL, et al. A look into the nature and causes of human errors in the intensive care unit. Crit Care Med. 1995;23(2):294-300. https://doi.org/10.1097/00003246-199502000-00015.

26. De Dreu CK, Weingart LR. Task versus relationship conflict, team performance, and team member satisfaction: a meta-analysis. J Appl Psychol. 2003;88(4):741. https://doi.org/10.1037/00219010.88.4.741.

27. Yeow JA, Ng PK, Tan KS, Chin TS, Lim WY. Effects of stress, repetition, fatigue and work environment on human error in manufacturing industries. Appl Sci. 2014;14(24):3464-71. https://doi.org/10.3923/jas.2014.3464.3471.

28. Tevlin R, Doherty E, Traynor O. Improving disclosure and management of medical error-an opportunity to transform the surgeons of tomorrow. the surgeon. 2013;11(6):338-43. 
https://doi.org/10.1016/j.surge.2013.07.008.

29. Santell JP. Medication errors: experience of the United States Pharmacopeia (USP). Jt Comm J Qual Patient Saf. 2005;31(2):114-9. https://doi.org/10.1016/s1553-7250(05)31016-6.

30. Chang $\mathrm{Y}-\mathrm{H}$, Wang $\mathrm{Y}-\mathrm{C}$. Significant human risk factors in aircraft maintenance technicians. Saf Sci. 2010;48(1):54-62. https://doi.org/10.1016/j.ssci.2009.05.004.

31. Vališ D, Milazzo M, Ancione G, Brkic V. Risk. Reliability and Safety: Innovating Theory and Practice. Taylor \& Francis Group London; 2017.

32. Maule AJ, Hockey GRJ, Bdzola L. Effects of time-pressure on decision-making under uncertainty: changes in affective state and information processing strategy. Acta Psychol (Amst). 2000;104(3):283-301. https://doi.org/10.1016/S0001-6918(00)00033-0.

33. Szalma J, Hancock P, Quinn S, editors. A meta-analysis of the effect of time pressure on human performance. Proceedings of the Human Factors and Ergonomics Society Annual Meeting; 2008: SAGE Publications Sage CA: Los Angeles, CA. https://doi.org/10.1177/154193120805201944.

34. Kirwan B. A guide to practical human reliability assessment. CRC press; 1994.

\section{Figures}

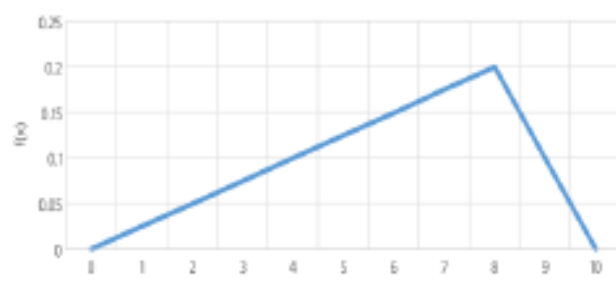

a) Triangular distribution ( $a=0 ; b=10 ; m=8$ ) for Avalable time.
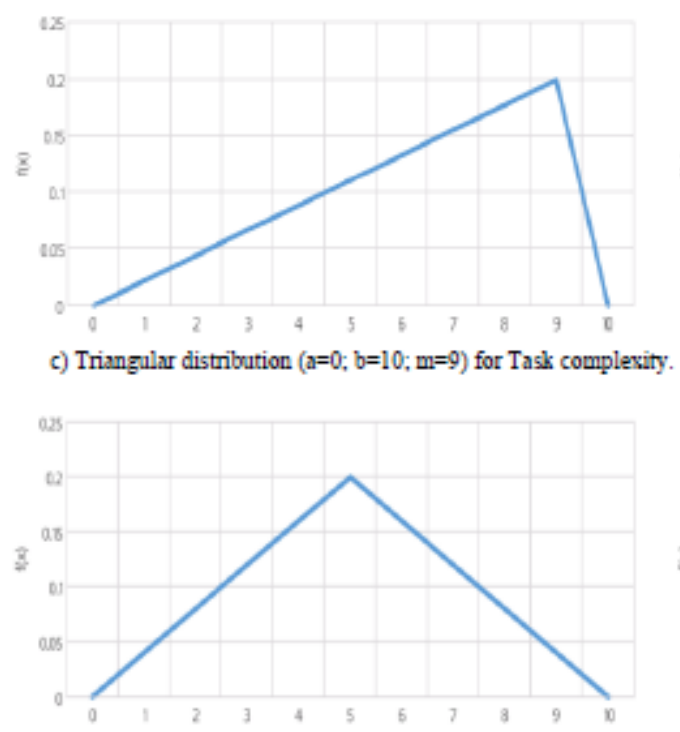

e) Triangular distribution ( $a=0 ; b=10 ; m=5$ ) for Teamwork; HMI and Procedures.

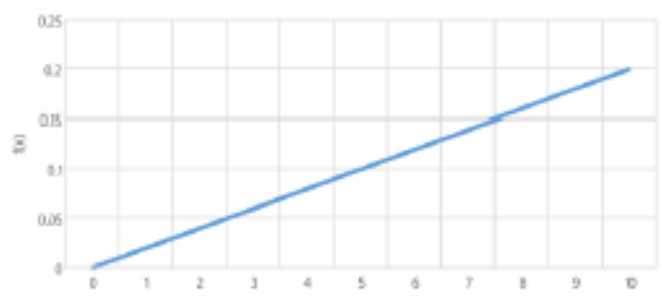

b) triangular distribution ( $\mathrm{a}=0, \mathrm{~b}=10 ; \mathrm{m}=0$ ) for Threat stress $\&$
Fatigue.

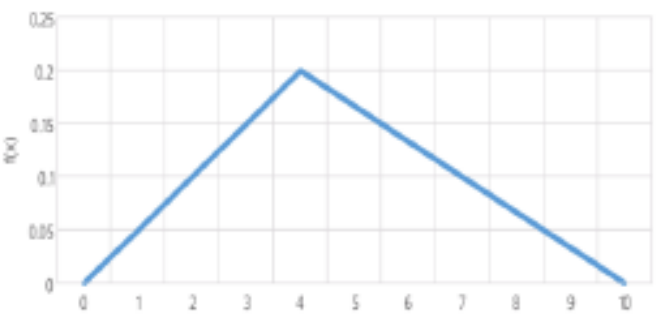

d) Triangular distribution ( $a=0$; $b=10 ; m=4$ ) for Experience/Training.

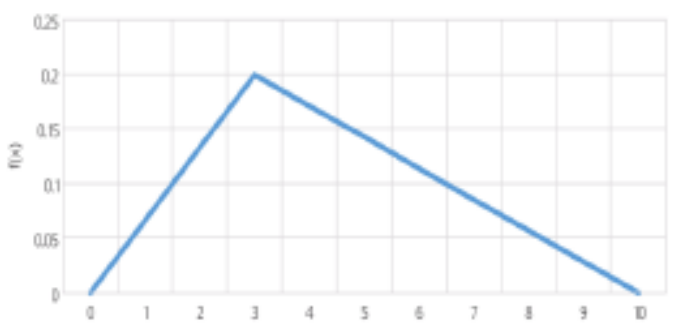

f) Triangular distribution ( $a=0, b=10 ; m=3$ ) for Working conditions

Figure 1 
$(a, b, c, d, e, f)$ Triangular distributions of the PSFs 